\title{
Probing the Dynamics of Neuronal Proteins In Vivo Using Non-Canonical Amino Acids Tagging
}

\author{
Kevin P. Lin ${ }^{1}$, Aya M. Saleh², Kathryn R. Jacobson ${ }^{2}$, Sarah Calve ${ }^{2}$ and Tamara L. \\ Kinzer-Ursem ${ }^{2}$ \\ ${ }^{1}$ Indiana University School of Medicine, ${ }^{2}$ Weldon School of Biomedical Engineering, \\ Purdue University, 206 South Martin Jischke Drive, West Lafayette, IN 47907
}

\section{Background and Hypothesis:}

More than 600 neurological disorders have been identified, each with varying degrees of complexity and level of molecular understanding. However, current approaches are inadequate to capture the complex progressive nature of most neurological diseases. Therefore, developing techniques capable of probing the temporal dynamics of neuronal proteins in rodents, the most commonly used experimental models, is imperative for proper understanding of mechanisms driving neurological disorders. In this project, a protein labeling technique that enables selective labeling of newly synthesized proteins in vivo is utilized. In this technique, the non-canonical amino acid azidohomoalanine (AHA) is injected into mice to achieve global proteome labeling. AHA is an azide-tagged methionine (Met) analog that is incorporated into the nascent proteins using endogenous translational mechanisms. The azide functional group of AHA allows selective enrichment of the newly synthesized proteins from brain tissues via click-chemistry using alkynebearing affinity tags. This will be followed by detecting the AHA-labeled protein using mass spectrometry. We hypothesize that this labeling technique will help map the dynamics of the brain proteome in health and disease. This will ultimately provide insights into mechanisms underlying complex neurological diseases.

\section{Experimental Design or Project Methods:}

C57BI/6 murine dams were injected with $0.1 \mathrm{mg} / \mathrm{g}$ AHA for two days. Brain tissues were harvested, homogenized and lysates were reacted with biotin-alkyne using copper-catalyzed click reaction. Biotinylated proteins were then enriched using NeutrAvidin beads and eluted by boiling in $2 \%$ SDS.

\section{Results:}

Tissues were fractionated into different subcellular components (cytosolic, nuclear, membrane, cytoskeletal, and extracellular matrix) using buffers of different stringency. Western blot analysis of clicked tissues using Streptavidin-fluorophore indicated effective incorporation of AHA into different cellular fractions of brain tissues. Additionally, the analysis of eluted proteins revealed successful enrichment and elution of AHA-labeled proteins.

\section{Conclusion and Potential Impact:}

Successful incorporation of AHA in nascent neuronal proteins can lead to a comprehensive quantitative approach for elucidating changes in the regulation of neuronal proteins in disease states. 